\title{
A Soybean Acyl Carrier Protein, GmACP, Is Important for Root Nodule Symbiosis
}

\author{
Jun Wang, ${ }^{1,2}$ Katalin Tóth, ${ }^{2}$ Kiwamu Tanaka, ${ }^{2}$ Cuong T. Nguyen, ${ }^{2}$ Zhe Yan, ${ }^{2}$ Laurent Brechenmacher, ${ }^{2}$ \\ Jeremy Dahmen, ${ }^{2}$ Mingjie Chen, ${ }^{3}$ Jay J. Thelen, ${ }^{3}$ Lijuan Qiu, ${ }^{1}$ and Gary Stacey ${ }^{2,3}$ \\ ${ }^{1}$ Key Laboratory of Crop Germplasm Utilization, Ministry of Agriculture/Institute of Crop Science, Chinese Academy of \\ Agricultural Sciences, Beijing, 100081, China; ${ }^{2}$ Division of Plant Sciences and National Center for Soybean Biotechnology, \\ and ${ }^{3}$ Division of Biochemistry, University of Missouri, Columbia 65211, U.S.A.
}

Submitted 23 September 2013. Accepted 18 December 2013.

Legumes (members of family Fabaceae) establish a symbiotic relationship with nitrogen-fixing soil bacteria (rhizobia) to overcome nitrogen source limitation. Single root hair epidermal cells serve as the entry point for bacteria to infect the host root, leading to development of a new organ, the nodule, which the bacteria colonize. In the present study, the putative role of a soybean acyl carrier protein (ACP), GmACP (Glyma18g47950), was examined in nodulation. ACP represent an essential cofactor protein in fatty acid biosynthesis. Phylogenetic analysis of plant ACP protein sequences showed that GmACP was classified in a legume-specific clade. Quantitative reverse-transcription polymerase chain reaction analysis demonstrated that $G m A C P$ was expressed in all soybean tissues but showed higher transcript accumulation in nodule tissue. RNA interferencemediated gene silencing of $G m A C P$ resulted in a significant reduction in nodule numbers on soybean transgenic roots. Fluorescent protein-labeled GmACP was localized to plastids in planta, the site of de novo fatty acid biosynthesis in plants. Analysis of the fatty acid content of root tissue silenced for GmACP expression, as determined by gas chromatography-mass spectrometry, showed an approximately $22 \%$ reduction, specifically in palmitic and stearic acid. Taken together, our data provide evidence that GmACP plays an important role in nodulation.

Root nodule symbiosis (RNS) is a conserved interaction between legume species and rhizobia that results in formation of a new organ, the nodule. Within the nodules, under stringent oxygen-limited conditions, atmospheric nitrogen is fixed and converted into ammonia by symbiotic bacteria, supplementing the host plant with a nitrogen source. Recognition of the symbiont by the plant host triggers two different processes occur-

J. Wang and K. Tóth contributed equally to this work.

Current address for Z Yan: Department of Molecular Biology and Genetics, Aarhus University, Aarhus, Denmark.

Current address for L. Brechenmacher: Health Sciences Centre, Department of Biochemistry and Molecular Biology, University of Calgary, Alberta, Canada.

Corresponding authors: L. Qui; E-mail: qiulijuan@caas.cn; and G. Stacey staceyg@missouri.edu

* The $\boldsymbol{e}$-Xtra logo stands for "electronic extra" and indicates that four supplementary figures and one supplementary table are published online.

(C) 2014 The American Phytopathological Society ring in parallel: bacterial infection and nodule development. Root hair cell curling entraps the rhizobia attached at the tip of the cell. An infection thread (IT) of plant origin is formed by which the bacteria exit the root hair cell and gain access to the root cortex. In advance of the growing IT, newly divided cortical cells expand to form the nodule primordia, subsequently giving rise to a nodule harboring the symbiotic bacteria. Bacteria from the IT are released into the cytosol of the infected cell, where they are encapsulated by a plant-derived membrane and form the so-called symbiosome (Roth et al. 1988).

In order to understand the processes behind rhizobial infection and nodule development, a number of studies have identified genes, proteins, and metabolites regulated during the interaction between legume host species and rhizobia (Benedito et al. 2008; Brechenmacher et al. 2008, 2009, 2010; Desbrosses et al. 2005; Libault et al. 2010a; Lim et al. 2010; Lohar et al. 2006; Oehrle et al. 2008; Wan et al. 2005). These studies have implicated a large number of genes, proteins, and metabolites involved in signal transduction, plant defense, carbon and nitrogen metabolism, and nutrient exchange during establishment of RNS.

In previous metabolomic profiling studies performed on Lotus japonicus tissues, including nodules (Colebatch et al. 2004; Desbrosses et al. 2005), and on soybean root hairs (Brechenmacher et al. 2010), different fatty acids (FA) were shown to accumulate in nodules (e.g., palmitic and stearic acid in L. japonicus nodules) relative to root tissue, and the levels of these compounds were strongly affected by rhizobial inoculation. These data suggest an important role for FA in the formation of RNS.

FA represent a significant source of carbon and essential components of different membranes as a part of membrane lipids. An earlier study estimated the surface area of newly formed membranes needed for IT growth, and symbiosomemembrane (i.e., membrane surrounding the intracellular rhizobial symbiont) formation is approximately $21,500 \mu^{2}$ per infected root nodule cell (Roth and Stacey 1989). FA that were found to be present in the symbiosome membrane represent saturated (palmitic acid and stearic acid) as well as unsaturated (palmitoleic acid, oleic acid, linoleic acid, and linolenic acid) FA (Gaude et al. 2004).

FA have a further function as components of surface layers; for example, as part of wax or cutin (Harwood 1996). Recent reports have suggested that FA and their derivatives are also involved in plant defense responses (Kachroo and Kachroo 2009; Kachroo et al 2004; Yaeno et al. 2004). One of the bestknown examples is the FA-derivative jasmonic acid, a phytohormone that has an essential role in wound signaling, plant- 
pathogen interactions, ultraviolet light stress, and insect attack (Weber 2002). Specific FA were also shown to modulate the responses to abiotic stresses (Kachroo and Kachroo 2009; Yaeno et al. 2004).

In plants, de novo FA biosynthesis occurs primarily in the plastid. The committed step is catalyzed by acetyl-CoA carboxylase (ACCase) to produce malonyl-CoA required for initiation and elongation of the nascent acyl moiety. These reactions are carried out by a series of enzymes sometimes referred to as the FA synthase (FAS), a putative multiprotein complex (Harwood
1988, 2005). In dicots and nongraminaceous monocots, both ACCase and FAS reactions are catalyzed by a series of proteins similar to the bacterial pathway (O'Hara et al. 2002; Sasaki et al. 1995). However, in graminaceous monocots, plastid ACCase is a homomeric enzyme in which all catalytic components are in tandem, forming a large polypeptide, orthologous to yeast and animal ACCase. A key component of the FAS is acyl carrier protein (ACP) which serves as a cofactor protein containing a phosphopantetheine prosthetic group, activated by ACP synthase (ACPS) at a conserved Asp-Ser-Leu (DSL) conserved motif, before be-

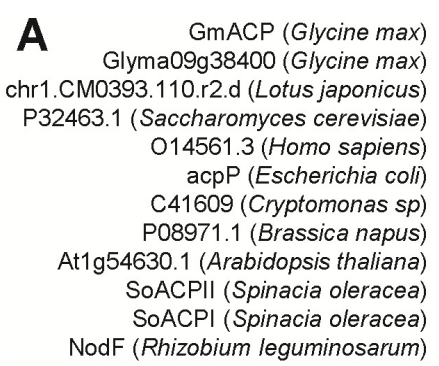

GmACP (Glycine max) Glyma09g38400 (Glycine max) chr1.CM0393.110.r2.d (Lotus japonicus) P32463.1 (Saccharomyces cerevisiae) 014561.3 (Homo sapiens) acpP (Escherichia coli) C41609 (Cryptomonas sp) P08971.1 (Brassica napus) At1g54630.1 (Arabidopsis thaliana) SoACPII (Spinacia oleracea)

SoACPI (Spinacia oleracea) NodF (Rhizobium leguminosarum)
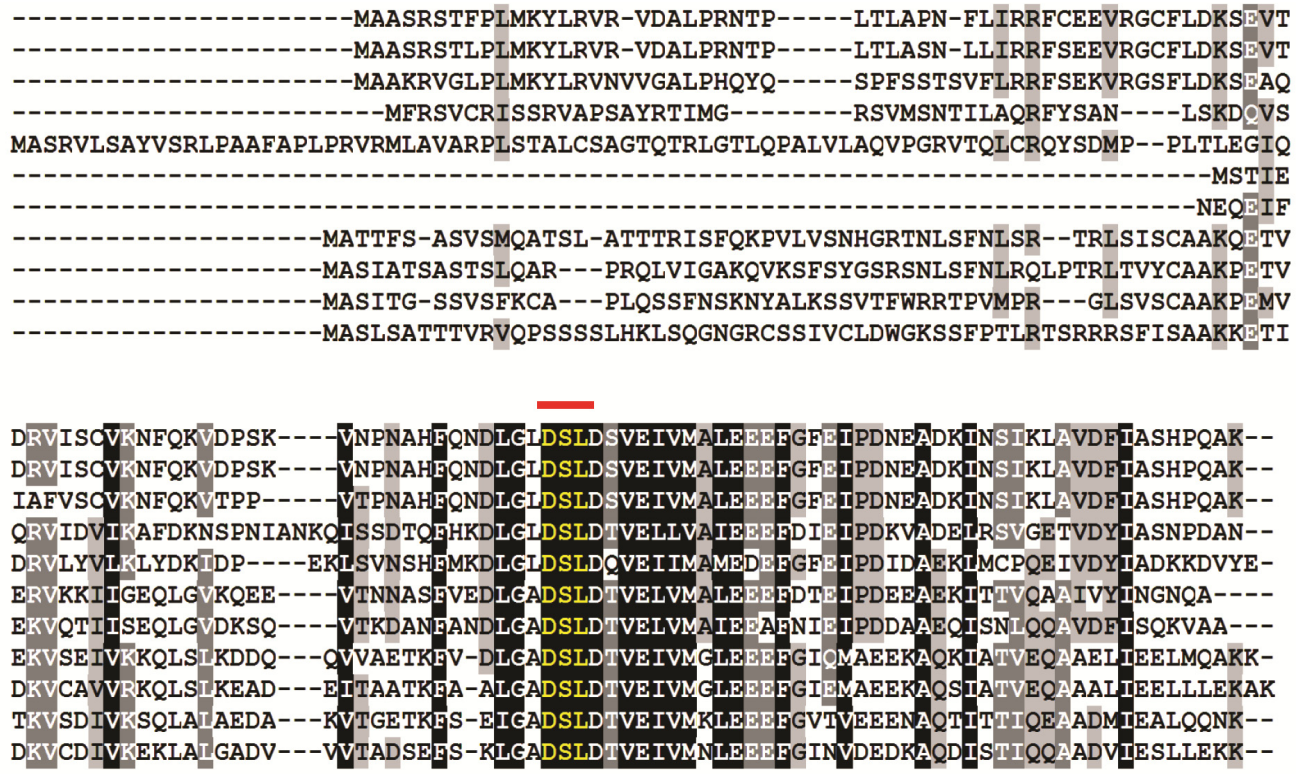

B

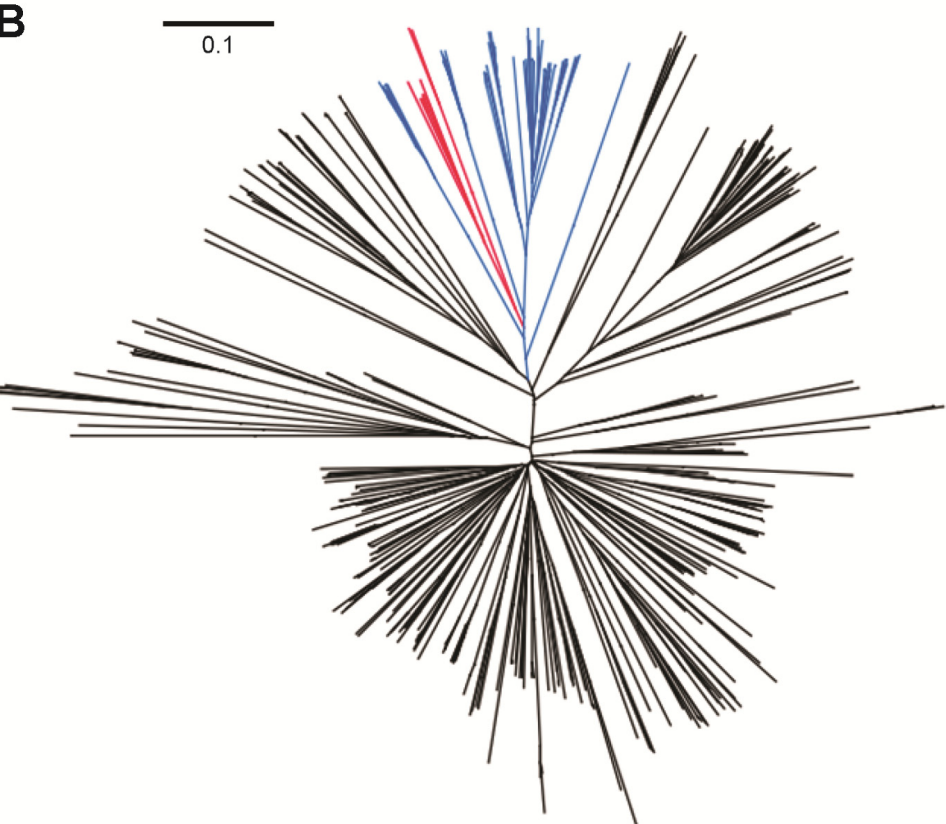

C

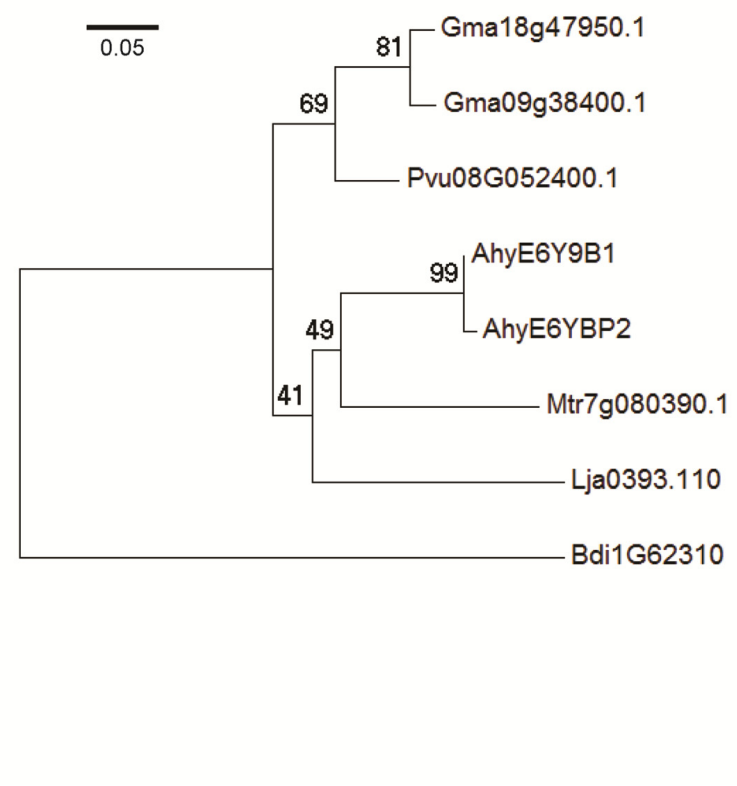

Fig. 1. Multiple alignment of acyl carrier protein (ACP) sequences and phylogenetic analysis. A, ACP amino acid sequences from different species across kingdoms were aligned. Amino acids underlined in red represent the conserved Asp-Ser-Leu (DSL) motif containing the highly conserved serine residue characteristic for ACP proteins across all kingdoms. Protein sequences involved are listed as follows: BnACP (Brassica napus, P08971.1); Csp. ACP (Cryptomonas sp., C41609); SoACPI (Spinacia oleracea, AAA34023.1); SoACPII (S. oleracea, P23235.1); acpP (Escherichia coli, AAB27925.2); ScACP (Saccharomyces cerevisiae, P32463.1); GmACP (Glycine max, Glyma18g47950.1); Glyma09g38400.1 (G. max); AtACP3 (Arabidopsis thaliana, At1g54630); HsACP (Homo sapiens; O14561.3); NodF (Rhizobium leguminosarum, YP_770461.1). B, Initial phylogenetic tree generated by neighbor-joining method (without bootstrap replication) using 517 ACP proteins. GmACP grouped to a legume-specific subclade (highlighted in red) within a clade with its closest homologs (highlighted in blue). C, Legume-specific subclade of GmACP (red highlighted in B). The tree was reconstructed using 54 ACP sequences from the homologous clade of GmACP (blue highlighted in B) and rooted using a Brachypodium distachyon ACP (XP_003557904.1). A tree with the highest log likelihood (-961.05) is shown. The percentage of trees in which the associated taxa clustered together is shown below the branches. The tree is drawn to scale, with branch lengths measured in the number of substitutions per site. All positions containing gaps and missing data were eliminated. There were 126 positions in the final dataset. 
ing able to accept acyl chains through a thioester bond (Chan and Vogel 2010; Safford et al. 1988).

ACP are small acidic proteins with the above-mentioned conserved phosphopantetheinyl-serine residue-contained DSL motif across all kingdoms. In plants, ACP are expressed both constitutively and in a tissue-specific manner (Alenka et al. 1992; Battey and Ohlrogge 1990; Bonaventure and Ohlrogge 2002; Guerra et al. 1986). In the model organism Arabidopsis thaliana, three ACP isoforms were identified in mitochondria and five ACP were localized to plastids (Harwood 2005). A similar distribution was observed in soybean (Ohlrogge and Kuo 1985), barley (Hordeum vulgare) (Hansen and Kauppinen 1991; Høj and Svendsen 1984), castor bean (Ricinus communis), cigar flower (Cuphea lanceolata) (Kopka et al. 1993), and rapeseed (Brassica napus) (Safford et al. 1988). In spinach, ACPI was present in dark-grown leaves whereas, in spinach seed and roots, only ACPII was detectable (Ohlrogge and Kuo 1985). The multiple ACP isoforms can also respond differently to environmental changes. For example, Arabidopsis ACP4 was induced by light, whereas the abundance of ACP2 or ACP3 was independent of light treatment (Bonaventure and Ohlrogge 2002). Strong ectopic expression of an Arabidopsis seed-predominant ACP in leaves altered the 18:3 FA and 16:3 FA compositions significantly (Branen et al. 2001).

Given the large need for membrane biogenesis during symbiotic development, as well as the potential signaling roles for FA, we were intrigued by the potential of ACP proteins to play an important role during soybean nodulation. Therefore, we focused our attention on GmACP (Glyma18g47950), which displayed a higher transcript accumulation in nodules 4 weeks postinoculation (wpi). We analyzed 517 putative ACP proteins from 43 plant species. Phylogenetic analysis showed that GmACP grouped with ACP proteins identified from other legume species, suggesting a legume-specific subclade. We confirmed that GmACP was localized to plastids in planta, suggesting a possible role in basal FA synthesis. Moreover, RNA interference (RNAi)-dependent gene silencing of GmACP expression resulted in reduced nodule numbers on inoculated transgenic soybean roots. Analysis of the FA content of GmACP-RNAi transgenic tissue, determined by gas chromatography-mass spectrometry (GC-MS), showed a reduction in total FA content in the $G m A C P$-RNAi root tissue, primarily due to a reduction in palmitic and stearic acids. Our results suggest that GmACP is important for nodulation via its function in FA biosynthesis.

\section{RESULTS}

The $G m A C P$ gene is a member of a well conserved, multigene family.

ACP proteins are well conserved among all kingdoms and, therefore, we took advantage of the recently sequenced soybean genome (Schmutz et al. 2010) to identify putative soybean ACP genes by sequence similarity (Supplementary Table S1). Subsequently, we chose the GmACP (Glyma18g47950) sequence and performed multiple protein sequence alignments using known ACP proteins from other species, in order to confirm that the identified soybean proteins belong to the ACP multigene family. A crucial criterion was to identify the well-known, highly conserved DSL motif in each ACP sequence (Supplementary Fig. S1). This motif is responsible for recognition by phosphopantetheinyltransferase, which is essential to activate ACP before accepting an acyl group (Mofid et al. 2002). Alignments included ACP proteins previously characterized from bacteria, yeast, cryptomonas, human, rat, rapeseed, spinach, soybean, $L$. japonicus, and Arabidopsis (Fig. 1A).

In order to perform a comprehensive phylogenetic analysis, we collected amino acid sequences of identified GmACP homo- logs in different plant species. Finally, 517 putative ACP from 43 plant species were identified and used, together with the 25 homologs from soybean, to construct an initial phylogenetic tree (without bootstrap replications) using the neighbor-joining method (Fig. 1B). This analysis identified a subclade where two soybean ACP proteins, GmACP (Glyma18g47950) and another homolog (Glyma09g38400), clustered together with putative ACP identified from different legumes, suggesting a legumespecific subclade (Fig. 1C; Supplementary Fig. S2). We reconstructed the tree using the maximum-likelihood method and Jones-Taylor-Thornton (JTT) substitution model (Jones et al. 1992), selecting 72 ACP from the clade where GmACP grouped. The newly built tree confirmed the formation of a subclade with legume-specific ACP-like proteins (Fig. 1C). This result was a further indication that ACP proteins might play an important role in the legume-rhizobia symbiosis.

\section{GmACP shows a higher transcript accumulation in nodule tissue.}

It was previously reported that ACP have different isoforms that can be expressed in a tissue-specific fashion (Battey and Ohlrogge 1990; Branen et al. 2001). In order to see whether the two soybean ACP proteins mentioned above, found in the same subclade, showed a similar expression pattern, we performed tissue-specific transcript analysis of GmACP and Glyma09g38400. Transcript abundance was analyzed by quantitative real-time reverse-transcription polymerase chain reaction (qRT-PCR) using total RNA isolated from the following soybean tissues: root, nodules, leaf, stem, and shoot tip (Fig. 2A). Glyma09g38400 displayed a generally higher expression level than $G m A C P$ in all tissues examined. However, GmACP showed approximately fourfold higher transcript accumulation in nodules 4 wpi with the soybean symbiont Bradyrhizobium japonicum in comparison with control uninoculated roots and also in comparison with roots separated from the nodules (Fig. 2B).

\section{Silencing of $G m A C P$ reduces nodule formation.}

The specific grouping of GmACP with other legume-specific $\mathrm{ACP}$, as well as the increased expression in nodules, are consistent with the hypothesis that ACP play an important role in nodulation and a subgroup may have evolved to provide this function. In order to specifically test the role of GmACP in nodulation, we used Agrobacterium rhizogenes-mediated gene transfer to express a $G m A C P$-RNAi construct in soybean transgenic roots. As a control, plant roots were also transformed with a vector expressing an RNAi construct specifically targeted to $\beta$-glucuronidase (GUS) (GUS-RNAi), an enzyme lacking in plants. Transgenic roots could be identified by the green fluorescent protein (GFP) marker expressed by the binary vector (Fig. 3C). The total number of nodules formed on the transgenic roots was counted on 34 and 50 plants transformed with either the GmACP-RNAi or GUS-RNAi construct, respectively. The number of nodules formed on the GmACP-RNAi transgenic roots was approximately $45 \%$ that of the control $(3.5 \pm 1.3$ and $7.6 \pm 2.8$ per transgenic root respectively, with $P<0.02$ ) (Fig. 3A). While taking advantage of the GFP marker, we had a look at sectioned nodules. They did not show obvious structural changes, regardless of the reduction in nodule number on transgenic roots (Supplementary Fig. S3).

In order to evaluate the efficiency of RNAi silencing, we quantified the expression level of GmACP mRNA in three representative transgenic roots. The GmACP expression level was reduced by $80 \%$ in comparison with the GUS-RNAi control (Student's $t$ test, $P<0.05$ ) (Fig. 3B). The expression level of the paralogous Glyma09g38400 gene showed significantly increased expression (Supplementary Fig. S4), as if compensating for the loss of GmACP expression. 


\section{Subcellular localization of GmACP.}

Plant ACP proteins have been localized to mitochondria, as well as plastids, representing the sites of de novo FA biosynthesis. GmACP was predicted to be localized to the plastid using WoLF pSORT, a general eukaryotic localization prediction tool (Horton et al. 2007). This prediction was in agreement with the lack of a mitochondrial targeting signal using SignaIP (Petersen et al. 2011). In order to experimentally confirm these computational predictions, we constructed a translational fusion of GmACP with GFP under a constitutive promoter and expressed this fusion protein in Nicotiana benthamiana leaves using A. tumefaciens-mediated gene transfer. The GmACP::GFP signal co-localized with a mCherry signal coming from a known, plastid marker protein (mCherry tagged 79-amino-acid small subunit of $N$. benthamiana RUBISCO, made in house), indicating plastid localization in the $N$. benthamiana leaf epidermal cells (Fig. 4). The green fluorescence signal showed an interesting dot-like pattern (accumulation) of the fusion protein, perhaps within the plastids. These data are consistent with the in silico predictions that GmACP displays plastidial localization, suggesting a possible role for this protein in de novo FA synthesis.

\section{Silencing of $G m A C P$ reduces palmitic and stearic acids.}

A previous study examined the lipid composition of the symbiosome membrane of soybean (Glycine max 'Stevens') nodules. The symbiosome membrane is composed of palmitic acid, stearic acid, palmitoleic acid, oleic acid, linoleic acid, and $\alpha$-linolenic acid (Gaude et al. 2004). As mentioned above, FA were reported to accumulate in L. japonicus nodules (palmitic and stearic acid) compared with roots. To clarify whether the observed nodulation phenotype caused by GmACP silencing is due to defective FA biosynthesis, the level of seven FA were measured by GC-MS in transgenic roots expressing either the GmACP-RNAi or GUS-RNAi control construct. In this assay, transgenic nodules and roots $0.5 \mathrm{~cm}$ flanking the nodules were isolated 4 to 6 wpi. We calculated the content of seven FA—namely, palmitic acid (16:0), stearic acid (18:0), oleic acid (18:1), linoleic acid (18:2), linolenic acid (18:3) eicosanoic acid (20:0), and docosanoic acid (22:0) - normalized to the fresh weight of the tissue used for the GC-MS analysis. We found that palmitic acid (16:0) content was significantly reduced approximately $21.9 \%$ while stearic acid (18:0) content was also significantly reduced approximately $21.8 \%$ in GmACP-RNAi-expressing roots but the levels of these two FA were not reduced in nodules (Fig. 5).

The FA composition of transgenic roots and nodules-specifically, palmitic acid (16:0), stearic acid (18:0), oleic acid (18:1), linoleic acid (18:2), and linolenic acid (18:3)—matched closely previous reports on the lipid composition of soybean roots and nodules (Gaude et al. 2004). On the other hand, we identified arachidic acid (eicosanoic, 20:0) and behenic acid (docosanoic, 22:0), which were not mentioned in previous studies. As for other soybean tissues, it was shown that soybean seed contain both arachidic and behenic acid (Silva et al. 2013). Another study that analyzed cellular FA of different rhi-
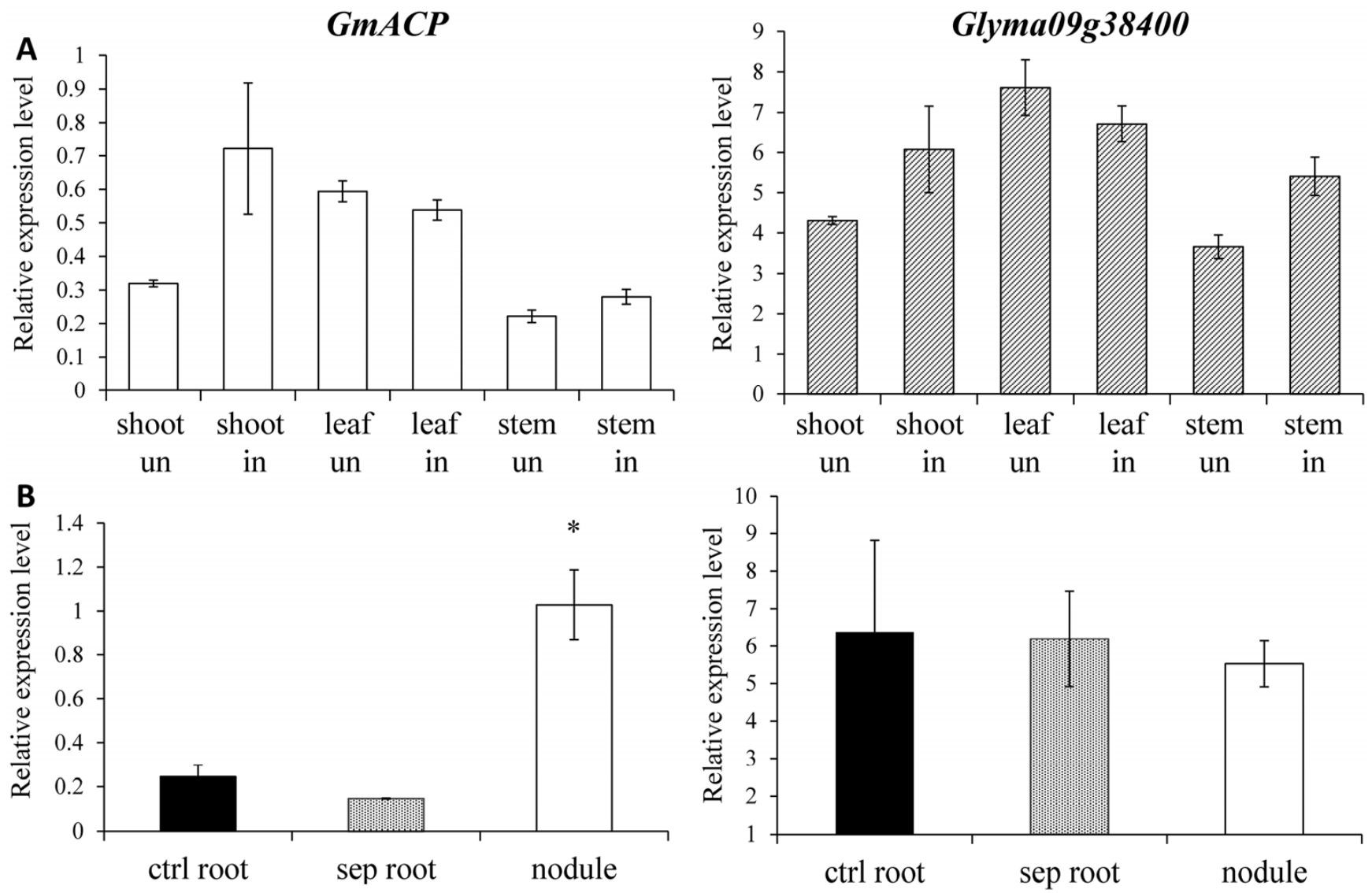

Fig. 2. Expression profile of $G m A C P$ in different soybean tissues. A, Relative gene expression level of $G m A C P$ and its putative paralog $G l y m a 09 g 38400$ in different aerial soybean tissues. B, Expression of GmACP and Glyma09g38400 in soybean roots and nodules 4 weeks postinoculation after Bradyrhizobium japonicum inoculation. The expression level was quantified by quantitative reverse-transcription polymerase chain reaction and normalized against soybean reference genes cons 4 and cons6 (Libault et al. 2008). Note that GmACP displayed an approximately fourfold increase in transcript abundance in nodules compared with control roots or roots separated from the nodules. The expression level of the GmACP paralog, Glyma09g38400, showed the same relative abundance in roots and nodules. The mean of three replications is shown. Error bars represent the standard error of the mean; $*$ indicates $P$ value $<0.05$ (Student's $t$ test). Abbreviations: un $=$ uninoculated, in $=$ inoculated, ctrl root $=$ uninoculated control root, sep root $=$ root separated from nodules. 
zobia could not detect arachidic acid in most of the analyzed rhizobia, including B. japonicum (Tighe et al. 2000). This study did not mention behenic acid. Therefore, we assume, at least for the case of arachidic acid, that this was synthesized by the plant host.
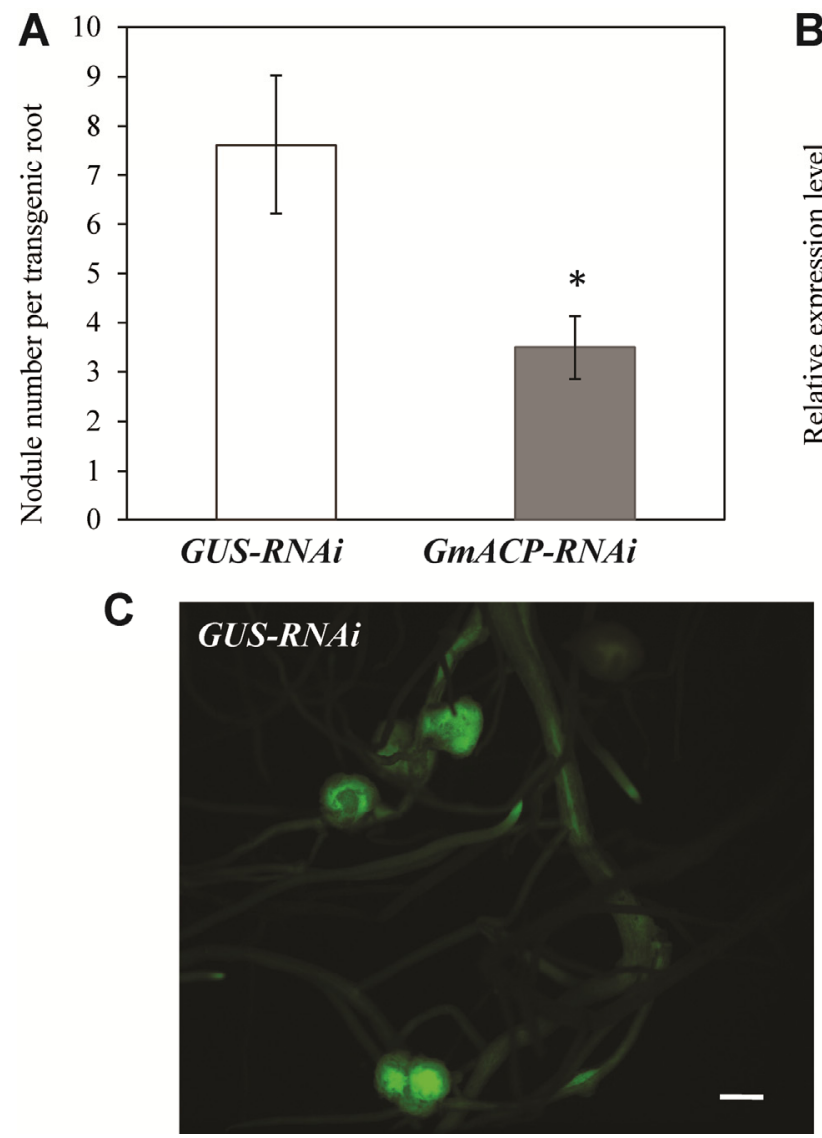

\section{DISCUSSION}

During establishment of the RNS, the host legume plant has to generate an estimated 35 -fold more membrane in comparison with a basic, nonsymbiotic lifestyle (Roth and Stacey 1989).
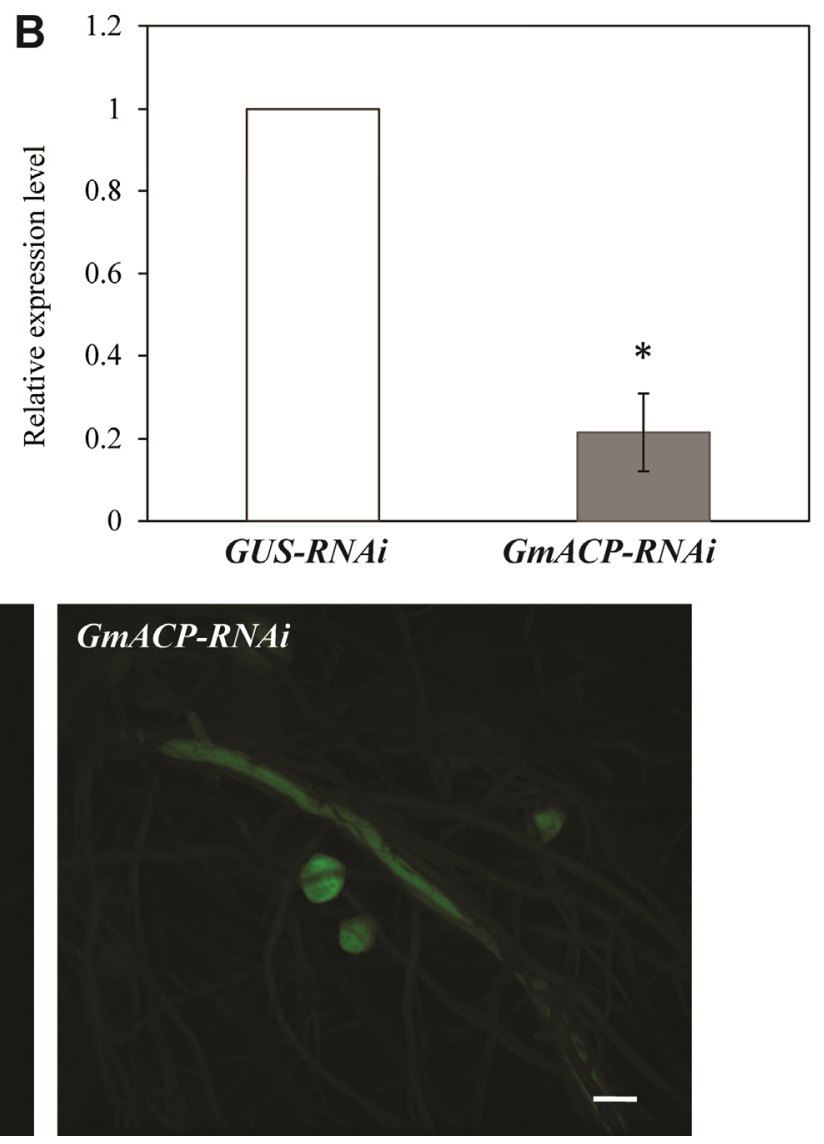

Fig. 3. Silencing of $G m A C P$ resulted in reduced nodule number. A, Comparison of the mean nodule numbers on transgenic roots identified by constitutive expression of a green fluorescent protein marker. Nodule numbers were counted on 50 and 34 roots transformed with the control $\beta$-glucuronidase RNA interference (GUS-RNAi) construct (white bar) and the GmACP-RNAi construct (gray bar), respectively. Standard error bars are shown; * indicates $P$ value < 0.02 (Student's $t$ test) was obtained when comparing both sets of roots. B, Measurement of mRNA abundance by quantitative reverse-transcription polymerase chain reaction for GmACP in GUS-RNAi and GmACP-RNAi roots. Gene expression levels were normalized against the soybean reference genes cons 4 and 6; * indicates $P$ value $<0.05$ (Student's $t$ test). C, Representative transgenic root and nodules expressing GUS-RNAi and GmACP-RNAi constructs. Scale bars represent $0.2 \mathrm{~cm}$.
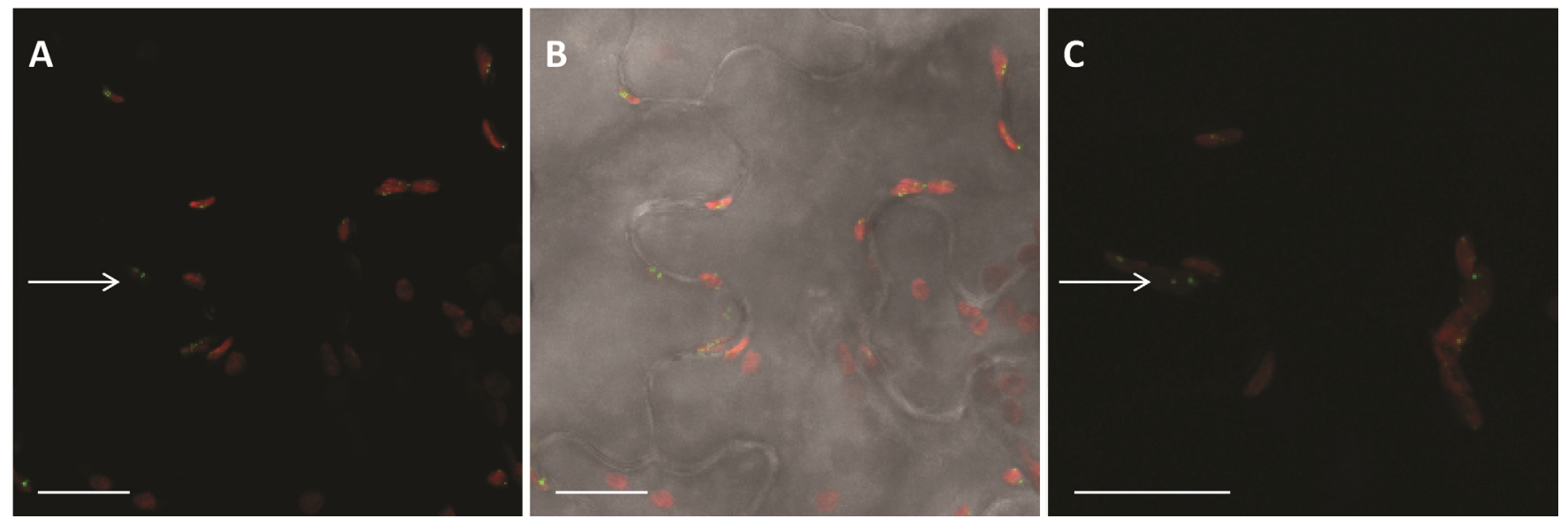

Fig. 4. GmACP shows plastid localization in planta. Subcellular localization of GmACP fused to the green fluorescent protein (GFP) fluorophore was investigated in planta using Nicotiana benthamiana leaf epidermal cells. Green fluorescent dots originating from GFP-tagged acyl carrier protein (ACP) (white arrow) can be observed, which co-localize with a mCherry-labeled plastid marker protein (79 amino acids of small RUBISCO subunit fused to mCherry red fluorescent protein). A, Overlay of GFP and mCherry; B, overlay of bright-field showing a leaf epidermal cell with fluorophores; C, close-up on mCherrylabeled plastids where the GFP-labeled GmACP protein localized. Scale bars represent $20 \mu \mathrm{m}$. Confocal laser-scanning microscopy was performed 2 days postinoculation. 
Lipids represent one of the major components of plasma membranes as well as organelle membranes and, in nodules, must also enclose the growing IT and intracellular symbionts (i.e., symbiosome membrane). The key building blocks of lipids are FA and they were found in increased amounts in L. japonicus as well as in soybean upon inoculation with the compatible rhizobial symbiont (Brechenmacher et al. 2010; Colebatch et al. 2004; Desbrosses et al. 2005).

FA biosynthesis plays a central and essential role in all living organisms. FA and their derivatives fulfill different functions in plants, including as basic components of membranes, as energy storage components, as signaling molecules, and as structural components of surface layers (Harwood 2005). A key protein involved in plant FA biosynthesis is the ACP (Chan and Vogel 2010).

GmACP displays the features of an ACP protein. Sequence alignment with other ACP proteins from across all kingdoms identified the characteristic and highly conserved DSL motif (Fig. 1A), the recognition site for ACPS (Mofid et al. 2002). Phylogenetic analysis grouped GmACP with another soybean ACP protein, Glyma09g38400, within an apparent legumespecific subclade, suggesting a legume-specific function (e.g., nodulation) for these proteins (Fig. 1B and C). In order to see whether both proteins might be involved in nodulation, tissuespecific expression profiling was performed on soybean apical shoot, leaf, stem, root, and nodule tissues (Fig. 2). Both $A C P$ soybean genes (GmACP and Glyma09g38400) showed a similar expression pattern in most tissues (Fig. 2), with Glyma09g38400 expressed at a consistently higher level (Fig. 2). However, only the $G m A C P$ transcript showed an approximately fourfold higher level in nodules (4 wpi with B. japonicum) compared with root tissue (Fig. 2B).

Consistent with these results, silencing of $G m A C P$ expression by RNAi significantly reduced nodulation efficiency to $40 \%$ of the control (Fig. 3), indicating an important role of GmACP in nodule formation. Surprisingly, we could not observe obvious defective nodules that might be expected as a consequence of the reduced FA content in the GmACP-RNA $i$ roots. Because there are $25 \mathrm{ACP}$-like proteins in soybean, it is possible that one of these genes could be providing sufficient functional redundancy to support some nodulation in the GmACP-silenced roots. Given that RNAi silencing always results in only a partial reduction in gene expression, it is also possible that variation in the levels of silencing throughout the root may also allow some nodulation to occur. The expression of the other ACP gene, Glyma09g38400, showed significantly increased expression, suggesting that it also might partially compensate for the reduction in GmACP expression, giving rise to a few fully developed nodules.

$\mathrm{ACP}$ are key components of FA synthesis and the process in plants takes place in either plastids or mitochondria. The subcellular location of GmACP was determined using a GFPtagged protein. The results clearly support a plastid location for the GmACP protein (Fig. 4), as was predicted in silico. This result is consistent with other published observations that ACP proteins localize to the scene of FA synthesis that occurs in plastids or mitochondria (Li-Beisson et al. 2010; Ohlrogge and Browse 1995), and it also supports our hypothesis that GmACP is most likely involved in de novo FA biosynthesis in plastids. The reduced FA content in transgenic roots expressing $G m A C P$-RNAi also supports this assumption (Fig. 5). The fact that reduction in stearic (18:0) and palmitic acid (16:0) content was found is also consistent with GmACP functioning in plastids, because the main product of the mitochondrial FA synthesis are FA with chain lengths of $\leq 14$ carbons (Koo et al. 2005; Wada et al. 1997).

The profile of detected FA correlated well with data previously described by Gaude and associates (2004), derived from soybean roots and nodules. However, we also detected arachidic and behenic acids. We analyzed FA content based on fresh weight of the tissues used for GC-MS. The content of palmitic acid and stearic acid was significantly reduced (approximately $22 \%$ ) in inoculated transgenic roots carrying the GmACPRNAi construct in comparison with control roots (Fig. 5). However, similar changes were not found when nodules were analyzed separately (Fig. 5). The lack of a nodule-specific phenotype seems contrary to the measured increase in GmACP transcript accumulation in nodules $4 \mathrm{wpi}$. It is possible that
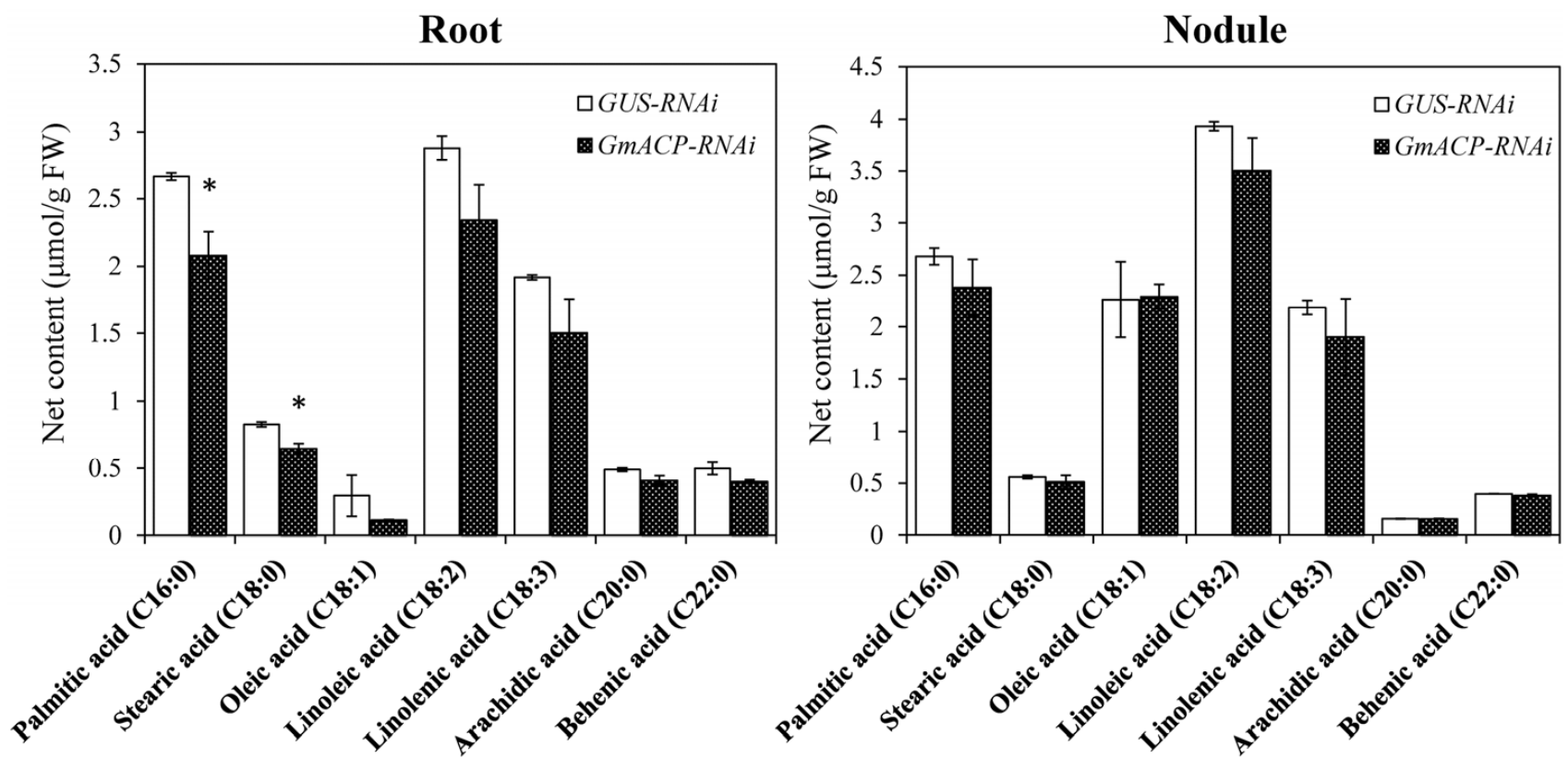

Fig. 5. Fatty acid content of transgenic soybean roots and nodules silenced for $G m A C P$ expression. Fatty acids were extracted and analyzed from control root tissue (expressing the $\beta$-glucuronidase [GUS] RNA interference [RNAi] construct) and roots expressing the GmACP-RNAi construct. Levels of palmitic and stearic acid were significantly reduced in root tissue silence for GmACP expression. However, such changes were not seen when nodule tissue was analyzed separately. Error bar indicates standard error of the mean. Two replications were performed for each experiment. 
root FA content may be the more important measure because increased FA synthesis may be more critical during nodule formation rather than later, when mature nodules are already formed (and analyzed by us for their FA content). RNAi silencing is never $100 \%$ effective and the nodules that did form on GmACP-RNAi plants showed normal nodule ultrastructure. It should also be emphasized that the expression level of the paralogous Glyma09g38400 gene was significantly increased in GmACP-RNAi transgenic roots in comparison with control transgenic roots. The expression level of Glyma09g38400 showed no change (root versus nodules) when tissue-specific expression profiling was performed (Fig. 2B). However, it is possible that the increase in Glyma09g38400 expression could partially compensate for the loss of $G m A C P$ and lessen or negate any changes in FA composition that may have occurred in the absence of this paralog. Furthermore, there is also the interesting possibility that the microsymbiont might be able to compensate for the lower fatty acid content. Indeed, Gaude and colleagues (Gaude et al. 2004) showed that stearic as well as palmitic acid are produced by the bacteria, and they showed a slightly higher content of these FA in bacteroids.

Given that these two FA are the main constituents of plasma membrane lipids, the reduction of the FA in the GmACP-RNAi transgenic roots is very likely affecting membrane biogenesis during nodulation. This corroborates the remarkable reduction of nodule numbers in the transgenic roots.

\section{MATERIALS AND METHODS}

\section{Alignment and phylogenetic analysis.}

We downloaded all available protein sequences from 42 plant genomes from the Phytozome (Goodstein et al. 2012) and Lotus databases for identifying all putative ACP. We also retrieved $16 \mathrm{ACP}$ sequences of Arachis hypogea from the Unipro database (Magrane and Consortium 2011) using the key words "acyl carrier protein" and "Arachis hypogea".

Experimentally characterized and manually annotated protein sequences of $163 \mathrm{ACP}$ (so-called seed sequences) from the PFAM database (Punta et al. 2011) with the PFAM family identifier PF00550 were used to create a profile-Hidden Markov Models (HMM) using the HMMer3 (Eddy 2011). This profileHMM model allowed us to search distantly related ACP homologs. The model was then used to search against protein sequences in each plant species to determine all putative ACP, with the E-value threshold set at $10^{-3}$ in order to capture all distantly related homologs.

We found 501 putative ACP in all 42 plant proteomes searched and we combined them with 16 A. hypogea ACP to generate an alignment using the ClustalOmega tool (Sievers et al. 2011). The alignment was then imported into the MEGA5.0 tool (Tamura et al. 2011) to create an initial neighbor-joining phylogenetic tree without bootstrap replications (Fig. 1B). We selected 72 ACP of the clade where GmACP grouped and reconstructed the phylogenetic tree again using the maximumlikelihood method (not shown), with 1,000 bootstrap replications and JTT substitution model (Jones et al. 1992).

\section{Plant growth, subcellular localization, and confocal laser- scanning microscopy.}

Soybean seed (G. $\max ($ L.) Merrill 'Williams 82') were germinated as described by Brechenmacher and associates (2009). Sterilized seed were planted into soil and grown in the greenhouse. Tissues of 2-week-old plants were collected for gene expression analysis 4 wpi with $B$. japonicum USDA 110.

Experiments to define the subcellular localization of GmACP in N. benthamiana leaves was performed as described by Tóth and associates (2012). Images were taken using a
Zeiss LSM 510 META NLO two-photon point-scanning confocal microscope with excitation wavelengths of $488 \mathrm{~nm}$ for GFP fluorophore and $543 \mathrm{~nm}$ for mCherry (Zeiss, Oberkochen, Germany). The water immersion objective lens HCX PL APO $20.0 \times 0.70$ IMM was used.

\section{RNA isolation, DNase treatment, and qRT-PCR.}

Total RNA from the various tissues used in this study were isolated by TRIzol (Ambion, Austin, TX, U.S.A.), followed by a DNase treatment (turbo DNase, Ambion), and then reverse transcribed into cDNA using M-MLV Reverse Transcriptase (Promega, Madison, WI, U.S.A.). Quantitative RT-PCR was performed as described by Libault and associates (2008). The geometric mean of cons 4 and cons6, encoding an ATP-binding cassette transporter and an F-box protein (Libault et al. 2008), were used as reference genes to normalize the expression levels. Primer set specificity was evaluated by a single dissociation curve profile of the amplicon for each qRT-PCR. This specificity was further confirmed by sequencing the PCR product and comparing back to the genomic sequence. The efficiency of each primer was quantified by LinRegPCR (Ramakers et al. 2003). The expression level of GmACP in different tissues was measured using at least three independent biological replicates. Data are reported as the mean value of these measurements. The comparison between samples was statistically validated using Student's $t$ test.

\section{Cloning.}

In order to silence $G m A C P$, a 144-bp fragment targeted to the $5^{\prime}$ untranslated region of $G m A C P$ was amplified from soybean cDNA. The amplified fragment was first cloned into pDONR/Zeo entry vector by BP clonase (GatewayBP Clonase II enzyme mix; Invitrogen, Carlsbad, CA, U.S.A.), and then the GmACP-RNAi-fragment was cloned into the Gatewaycompatible destination vector CGT11017A by LR reaction (GatewayLR Clonase II enzyme mix; Invitrogen) to form a hairpin structure. The vector used for silencing carries the gene encoding the GFP, driven by the strong Figwort mosaic virus promoter (Govindarajulu et al. 2008), thereby allowing the detection of transgenic roots by GFP expression as a marker. CGT5200 was used as the RNAi control vector for GmACPRNAi, as described previously (Govindarajulu et al. 2009; Libault et al. 2010b). This vector contains an RNAi construct directed against GUS. The integration and fidelity of these vector constructs were confirmed by sequencing. Finally, both RNAi and control vectors were transformed into Agrobacterium rhizogenes strain $\mathrm{K} 599$ by electroporation for hairy root transformation.

In order to investigate the subcellular localization of GmACP, the complete cDNA of GmACP was amplified using soybean cDNA and cloned into pDONR/Zeo Gateway-compatible entry vector. GmACP cDNA was fused to the $\mathrm{N}$ terminus of GFP in pMDC83 by LR clonase to make a translational fusion (Curtis and Grossniklaus 2003). All the constructs were verified by sequencing.

\section{Composite plant generation and nodulation assays.}

Soybean composite plants were generated according to previously published protocols using $A$. rhizogenes (Taylor et al. 2006). In order to generate transgenic soybean roots, the shoot above the first true leaves was cut and incubated with $4 \mathrm{ml}$ of an $A$. rhizogenes suspension (carrying the respective constructs; optical density at $\left.600 \mathrm{~nm}\left[\mathrm{OD}_{600}\right]=0.3\right)$ in rock wool cubes for 3 to 4 days (under ambient laboratory conditions). One week later, plants were transferred into a vermiculite and perlite mixture (3:1) and first grown in laboratory conditions; another week later, plants were moved to the greenhouse. Each 
plant was inoculated with $10 \mathrm{ml}$ of $B$. japonicum (USDA110) culture suspended in nitrogen-free plant nutrient solution $\left(\mathrm{OD}_{600}=0.08\right)$ approximately 1 week ( 7 days $)$ after the plants were returned to the greenhouse (Lullien et al. 1987). Nodulation phenotype was analyzed using a Leica MZFLIII stereoscope (GFPLF filter) 4 to 6 wpi. Only the nodules formed on transgenic roots, as determined by GFP marker, were counted. The data reported represent at least four independent experiments. Each experiment included at least 12 composite plants.

\section{FA composition determination.}

Quantification of the FA composition of nodulated roots was performed as described by $\mathrm{Wu}$ and colleagues (1994). A total of 15 to $150 \mathrm{mg}$ (fresh weight) of root tissue was isolated. This included all nodules and the root tissue $0.5 \mathrm{~cm}$ flanking each nodule (i.e., a $1-\mathrm{cm}$ root section). Tissues were heated at $80^{\circ} \mathrm{C}$ in a water bath in $3 \mathrm{ml}$ of $2.5 \%$ ( $\mathrm{vol} / \mathrm{vol}) \mathrm{H}_{2} \mathrm{SO}_{4}$ in methanol for $90 \mathrm{~min}$ in screw-capped tubes. Heptadecanoic acid (17:0) was used as internal standard for quantification. FA were extracted to the organic phase by shaking in $4.5 \mathrm{ml}$ of $0.9 \%$ $\mathrm{NaCl}$ solution and $1.5 \mathrm{ml}$ of hexane. After centrifugation at 3,000 rpm for $1 \mathrm{~min}$ to break emulsions formed during shaking, the organic phase was collected for GC analysis. GC analysis was performed on an Agilent 5973N GC-MS system equipped with a flame ionization detector and a DB-23 capillary column $(0.25 \mathrm{~mm}$ by $30 \mathrm{~m}$ by $0.25 \mu \mathrm{m})$. The temperature program applied was initiated at $150^{\circ} \mathrm{C}$ and followed by a $2^{\circ} \mathrm{C} / \mathrm{min}$ ramp-up to $200^{\circ} \mathrm{C}$ and holding for $5 \mathrm{~min}$. Split ratio in our case was 25:1. Helium was used as carrier gas and at an initial flow of $0.5 \mathrm{ml} \mathrm{min}{ }^{-1}$. Peak area of FA was calculated by Enhanced ChemStation (MSD ChemStation D.01.02.16.1s). FA data were imported into an Excel sheet and FA content $(\mu \mathrm{mol} / \mathrm{g})$ was calculated based on fresh weight of tissue used for GC-MS analysis. Variance was analyzed by Student's $t$ test.

\section{ACKNOWLEDGMENTS}

Research was funded by grants from the United States National Science Foundation Plant Genome program (grant number DBI-0421620) and the China National Transgenic Major Program on Cloning and Functional Verification of Genes Controlling Important Traits (grant number 2013ZX08009003) and was also supported by the Chinese Scholarship Council.

\section{LITERATURE CITED}

Alenka, H. R., Dusty, P. B., and Ohlrogge, J. B. 1992. Expression of constitutive and tissue-specific acyl-carrier protein isoforms in Arabidopsis. Plant Physiol. 98:206-214.

Battey, J. F., and Ohlrogge, J. B. 1990. Evolutionary and tissue-specific control of expression of multiple acyl-carrier protein isoforms in plants and bacteria. Planta 180:352-360.

Benedito, V. A., Torres-Jerez, I., Murray, J. D., Andriankaja, A., Allen, S., Kakar, K., Wandrey, M., Verdier, J., Zuber, H., Ott, T., Moreau, S., Niebel, A., Frickey, T., Weiller, G., He, J., Dai, X., Zhao, P. X., Tang, Y., and Udvardi, M. K. 2008. A gene expression atlas of the model legume Medicago truncatula. Plant J. 55:504-513.

Bonaventure, G., and Ohlrogge, J. B. 2002. Differential regulation of mRNA levels of acyl carrier protein isoforms in Arabidopsis. Plant Physiol. 128:223-235.

Branen, J. K., Chiou, T. J., and Engeseth, N. J. 2001. Overexpression of acyl carrier protein-1 alters fatty acid composition of leaf tissue in Arabidopsis. Plant Physiol. 127:222-229.

Brechenmacher, L., Kim, M. Y., Benitez, M., Li, M., Joshi, T., Calla, B., Lee, M. P., Libault, M., Vodkin, L. O., Xu, D., Lee, S. H., Clough, S. J., and Stacey, G. 2008. Transcription profiling of soybean nodulation by Bradyrhizobium japonicum. Mol. Plant-Microbe Interact. 21:631-645.

Brechenmacher, L., Lee, J., Sachdev, S., Song, Z., Nguyen, T. H., Joshi, T., Oehrle, N., Libault, M., Mooney, B., Xu, D., Cooper, B., and Stacey, G. 2009. Establishment of a protein reference map for soybean root hair cells. Plant Physiol. 149:670-682.

Brechenmacher, L., Lei, Z., Libault, M., Findley, S., Sugawara, M., Sadowsky, M. J., Sumner, L. W., and Stacey, G. 2010. Soybean metabo- lites regulated in root hairs in response to the symbiotic bacterium $\mathrm{Bra}$ dyrhizobium japonicum. Plant Physiol. 153:1808-1822.

Chan, D. I., and Vogel, H. J. 2010. Current understanding of fatty acid biosynthesis and the acyl carrier protein. Biochem. J. 430:1-19.

Colebatch, G., Desbrosses, G., Ott, T., Krusell, L., Montanari, O., Kloska, S., Kopka, J., and Udvardi, M. K. 2004. Global changes in transcription orchestrate metabolic differentiation during symbiotic nitrogen fixation in Lotus japonicus. Plant J. 39:487-512.

Curtis, M. D., and Grossniklaus, U, 2003. A gateway cloning vector set for high-throughput functional analysis of genes in planta. Plant Physiol. 133:462-469.

Desbrosses, G. G., Kopka, J., and Udvardi, M. K. 2005. Lotus japonicus metabolic profiling. Development of gas chromatography-mass spectrometry resources for the study of plant-microbe interactions. Plant Physiol. 137:1302-1318.

Eddy, S. R. 2011. Accelerated profile HMM searches. PLoS Comput. Biol. 7:e1002195.

Gaude, N., Tippmann, H., Flemetakis, E., Katinakis, P., Udvardi, M., and Dörmann, P. 2004. The galactolipid digalactosyldiacylglycerol accumulates in the peribacteroid membrane of nitrogen-fixing nodules of soybean and Lotus. J. Biol. Chem. 279:34624-34630.

Goodstein, D. M., Shu, S., Howson, R., Neupane, R., Hayes, R. D., Fazo, J., Mitros, T., Dirks, W., Hellsten, U., Putnam, N., and Rokhsar, D. S. 2012. Phytozome: A comparative platform for green plant genomics. Nucleic Acids Res. 40:D1178-D1186.

Govindarajulu, M., Elmore, J. M., Fester, T., and Taylor, C. G. 2008. Evaluation of constitutive viral promoters in transgenic soybean roots and nodules. Mol. Plant-Microbe Interact. 21:1027-1035.

Govindarajulu, M., Kim, S. Y., Libault, M., Berg, R. H., Tanaka, K., Stacey, G., and Taylor, C. G. 2009. GS52 ecto-apyrase plays a critical role during soybean nodulation. Plant Physiol. 149:994-1004.

Guerra, D. J., Ohlrogge, J. B., and Frentzen, M. 1986. Activity of acyl carrier protein isoforms in reactions of plant fatty acid metabolism. Plant Physiol. 82:448-453.

Hansen, L., and Kauppinen, S. 1991. Barley acyl carrier protein II: Nucleotide sequence of cDNA clones and chromosomal location of the Acl2 gene. Plant Physiol. 91:472-474.

Harwood, J. L. 1988. Fatty acid metabolism. Annu. Rev. Plant Physiol. 39:101-138.

Harwood, J. L. 1996. Recent advances in the biosynthesis of plant fatty acids. Biochem. Biophys. Acta 1301:7-56.

Harwood, J. L. 2005. Fatty acid biosynthesis. Pages 27-66 in: Plant Lipids: Biology, Utilization and Manipulation. D. J. Murphy, ed. Blackwell Publishing, Oxford.

Høj, P., and Svendsen, I. 1984. Barley chloroplasts contain two acyl carrier proteins coded for by different genes. Carlsberg Res. Commun. 49:483492.

Horton, P., Park, K. J., Obayashi, T., Fujita, N., Harada, H., Adams-Collier, C. J., and Nakai, K 2007. WoLF PSORT: Protein localization predictor. Nucleic Acids Res. 35:W585-587.

Jones, D. T., Taylor, W. R., and Thornton, J. M. 1992. The rapid generation of mutation data matrices from protein sequences. Comput. Appl. Biosci. 8:275-282.

Kachroo, A., and Kachroo, P. 2009. Fatty acid-derived signals in plant defense. Annu. Rev. Phytopathol. 47:153-176.

Kachroo, A., Venugopal, S. C., Lapchyk, L., Falcone, D., Hildebrand, D., and Kachroo, P. 2004. Oleic acid levels regulated by glycerolipid metabolism modulate defense gene expression in Arabidopsis. Proc. Natl. Acad. Sci. U.S.A. 101:5152-5157.

Koo, A. J., Fulda, M., Browse, J., and Ohlrogge, J. B. 2005. Identification of a plastid acyl-acyl carrier protein synthetase in Arabidopsis and its role in the activation and elongation of exogenous fatty acids. Plant $\mathbf{J}$. 44:620-632.

Kopka, J., Robers, M., Schuch, R., and Spener, F. 1993. Acyl carrier proteins from developing seeds of Cuphea lanceolata Ait. Planta 191:102 111.

Libault, M., Thibivilliers, S., Bilgin, D. D., Radwan, O., Benitez, M. Clough, S. J., and Stacey, G. 2008. Identification of four soybean reference genes for gene expression normalization. Plant Genome J. 1:44.

Libault, M., Farmer, A., Brechenmacher, L., Drnevich, J., Langley, R. J., Bilgin, D. D., Radwan, O., Neece, D. J., Clough, S. J., May, G. D., and Stacey, G. 2010a. Complete transcriptome of the soybean root hair cell, a single-cell model, and its alteration in response to Bradyrhizobium japonicum infection. Plant Physiol. 152:541-552.

Libault, M., Zhang, X. C., Govindarajulu, M., Qiu, J., Ong, Y. T. Brechenmacher, L., Berg, R. H., Hurley-Sommer, A., Taylor, C. G., and Stacey, G. 2010b. A member of the highly conserved FWL (tomato $F W 2.2$-like) gene family is essential for soybean nodule organogenesis. Plant J. 62:852-864.

Li-Beisson, Y., Shorrosh, B., Beisson, F., Andersson, M. X., Arondel, V., 
Bates, P. D., Baud, S., Bird, D., DeBono, A., Durrett, T. P., Franke, R. B., Graham, I. A., Katayama, K., Kelly, A. A., Larson, T., Markham, J. E., Miquel, M., Molina, I., Nishida, I., Rowland, O., Samuels, L., Schmid, K. M., Wada, H., Welti, R., Xu, C., Zallot, R., and Ohlrogge, J. 2010. Acyl-lipid metabolism. Arabidopsis Book 8:e0133.

Lim, C. W., Park, J. Y., Lee, S. H., and Hwang, C. H. 2010. Comparative proteomic analysis of soybean nodulation using a supernodulation mutant, SS2-2. Biosci. Biotechnol. Biochem. 74:2396-2404.

Lohar, D. P., Sharopova, N., Endre, G., Penuela, S., Samac, D., Town, C., Silverstein, K. A., and Van den Bosch, K. A. 2006. Transcript analysis of early nodulation events in Medicago truncatula. Plant Physiol. 140:221-234.

Lullien, V., Barker, D. G., Lajudie, P., and Huguet, T. 1987. Plant gene expression in effective and ineffective root nodules of alfalfa (Medicago sativa). Plant Mol. Biol. 9:469-478.

Magrane, M., and Consortium, U. 2011. UniProt Knowledgebase: A hub of integrated protein data. Database (Oxford) 2011:bar009. Published online.

Mofid, M. R., Finking, R., and Marahiel, M. A. 2002. Recognition of hybrid peptidyl carrier proteins/acyl carrier proteins in nonribosomal peptide synthetase modules by the 4'-phosphopantetheinyl transferases AcpS and Sfp. J. Biol. Chem. 277:17023-17031.

Oehrle, N. W., Sarma, A. D., Waters, J. K., and Emerich, D. W. 2008. Proteomic analysis of soybean nodule cytosol. Phytochemistry 69:24262438

O'Hara, P., Slabas, A. R., and Fawcett, T. 2002. Fatty acid and lipid biosynthetic genes are expressed at constant molar ratios but different absolute levels during embryogenesis. Plant Physiol. 129:310-320.

Ohlrogge, J., and Browse, J. 1995. Lipid biosynthesis. Plant Cell 7:957970

Ohlrogge, J. B., and Kuo, T. M. 1985. Plants have isoforms for acyl carrier protein that are expressed differently in different tissues. J. Biol. Chem. 260:8032-8037.

Petersen, T. N., Brunak, S., von Heijne, G., and Nielsen, H. 2011. SignalP 4.0: Discriminating signal peptides from transmembrane regions. Nat. Methods 8:785-786.

Punta, M., Coggill, P. C., Eberhardt, R. Y., Mistry, J., Tate, J., Boursnell, C., Pang, N., Forslund, K., Ceric, G., Clements, J., Heger, A., Holm, L., Sonnhammer, E. L. L., Eddy, S. R., Bateman, A., and Finn, R. D. 2011. The Pfam protein families database. Nucleic Acids Res. 40:D290-D301.

Ramakers, C., Ruijter, J. M., Deprez, R. H., and Moorman, A. F. 2003. Assumption-free analysis of quantitative real-time polymerase chain reaction (PCR) data. Neurosci. Lett. 339:62-66.

Roth, L. E., and Stacey, G. 1989. Bacterium release into host cells of nitrogen-fixing soybean nodules: The symbiosome membrane comes from three sources. Eur. J. Cell Biol. 49:13-23.

Roth, L. E., Jeon, K., and Stacey, G. 1988. Homology in endosymbiotic systems: The term "symbiosome". Pages 220-225 in: Molecular Genetics of Plant Microbe Interactions. R. Palacios and D. P. Verma, eds. American Phytopathological Society Press, St. Paul, MN, U.S.A.

Safford, R., Windust, J. H., Lucas, C., De Silva, J., James, C. M., Hellyer, A., Smith, C. G., Slabas, A. R., and Hughes, S. G. 1988. Plastid-localised seed acyl-carrier protein of Brassica napus is encoded by a distinct, nuclear multigene family. Eur. J. Biochem. 174:287-295.

Sasaki, Y., Konishi, T., and Nagono, Y. 1995. The compartmentation of acetyl-coenzyme a carboxylase in plants. Plant Physiol. 108:445-449.
Schmutz, J., Cannon, S. B., Schlueter, J., Ma, J., Mitros, T., Nelson, W., Hyten, D. L., Song, Q., Thelen, J. J., Cheng, J., Xu, D., Hellsten, U., May, G. D., Yu, Y., Sakurai, T., Umezawa, T., Bhattacharyya, M. K., Sandhu, D., Valliyodan, B., Lindquist, E., Peto, M., Grant, D., Shu, S., Goodstein, D., Barry, K., Futrell-Griggs, M., Abernathy, B., Du, J., Tian, Z., Zhu, L., Gill, N., Joshi, T., Libault, M., Sethuraman, A., Zhang, X. C., Shinozaki, K., Nguyen, H. T., Wing, R. A., Cregan, P., Specht, J., Grimwood, J., Rokhsar, D., Stacey, G., Shoemaker, R. C., and Jackson, S. A. 2010. Genome sequence of the palaeopolyploid soybean. Nature 463:178-183.

Sievers, F., Wilm, A., Dineen, D., Gibson, T. J., Karplus, K., Li, W., Lopez, R., McWilliam, H., Remmert, M., Söding, J., Thompson, J. D., and Higgins, D. G. 2011. Fast, scalable generation of high-quality protein multiple sequence alignments using Clustal Omega. Mol. Syst. Biol. 7:539. Published online.

Silva, L. R., Pereira, M. J., Azevedo, J., Mulas, R., Velazquez, E., GonzálezAndrés, F., Valentão, P., and Andrade, P. B. 2013. Inoculation with Bradyrhizobium japonicum enhances the organic and fatty acids content of soybean (Glycine max (L.) Merrill) seeds. Food Chem. 141:3636-3648.

Tamura, K., Peterson, D., Peterson, N., Stecher, G., Nei, M., and Kumar, S. 2011. MEGA5: Molecular evolutionary genetics analysis using maximum likelihood, evolutionary distance, and maximum parsimony methods. Mol. Biol. Evol. 28:2731-2739.

Taylor, C. G., Fuchs, B., Collier, R., and Lutke, W. K. 2006. Generation of composite plants using Agrobacterium rhizogenes. Methods Mol. Biol. 343:155-167.

Tighe, S. W., de Lajudie, P., Dipietro, K., Lindström, K., Nick, G., and Jarvis, B. D. 2000. Analysis of cellular fatty acids and phenotypic relationships of Agrobacterium, Bradyrhizobium, Mesorhizobium, Rhizobium and Sinorhizobium species using the Sherlock Microbial Identification System. Int. J. Syst. Evol. Microbiol. 50:787-801.

Tóth, K., Stratil, T. F., Madsen, E. B., Ye, J., Popp, C., Antolín-Llovera, M., Grossmann, C., Jensen, O. N., Schüssler, A., Parniske, M., and Ott, T. 2012. Functional domain analysis of the Remorin protein LjSYMREM1 in Lotus japonicus. PLoS One 7:e30817.

Wada, H., Shintani, D., and Ohlrogge, J. 1997. Why do mitochondria synthesize fatty acids? Evidence for involvement in lipoic acid production. Proc. Natl. Acad. Sci. U.S.A. 94:1591-1596.

Wan, J., Torres, M., Ganapathy, A., Thelen, J., DaGue, B. B., Mooney, B. Xu, D., and Stacey, G. 2005. Proteomic analysis of soybean root hairs after infection by Bradyrhizobium japonicum. Mol. Plant-Microbe Interact. 18:458-467

Weber, H. 2002. Fatty acid-derived signals in plants. Trends Plant Sci. 7:217-224.

Wu, J., James, D. W., Jr., Dooner, H. K., and Browse, J. 1994. A mutant of Arabidopsis deficient in the elongation of palmitic acid. Plant Physiol 106:143-150

Yaeno, T., Matsuda, O., and Iba, K. 2004. Role of chloroplast trienoic fatty acids in plant disease defense responses. Plant J. 40:931-941.

\section{AUTHOR-RECOMMENDED INTERNET RESOURCES}

Kazusa DNA Research Institute Lotus database: www.kazusa.or.jp/lotus Phytozome database: www.phytozome.net 